Zusammenfassung der Dissertation mit dem Titel

„Regulierungsgetriebene hybride Finanzierungsinstrumente ${ }^{66}$

Dissertation vorgelegt von Dominik Mohr

Erstgutachter: Prof. Dr. Christoph Kern

Zweitgutachter: Prof. Dr. Dirk Verse

Institut für ausländisches und internationales Privat- und Wirtschaftsrecht 


\title{
ZUSAMMENFASSUNG DER WESENTLICHEN ERGEBNISSE
}

\author{
Regulierungsgetriebene hybride Finanzierungsinstrumente
}

Die aktuelle Lage um die Corona-Pandemie trifft nicht nur die Bevölkerungen dieser Welt teilweise schwer, sie hat auch Auswirkungen auf die Bankenregulierung. In Zuge der wirtschaftlichen Rezession und dem damit einhergehenden Druck auf die Bankbilanzen hat die europäische Bankenaufsicht beschlossen, den Kreditinstituten mehr Zeit bei der Umsetzung der Eigenkapitalanforderungen zu geben. Nichtdestotrotz bedarf es selbst bis 2022 noch einiger Anstrengung auf Seiten der Banken, die Eigenkapitalquote auf das geforderte Maß hochzufahren. Deshalb stellen hybride Finanzierungsinstrumente in der Bankenregulierung nach wie vor ein hochaktuelles Thema dar, zumal das Emissionsvolumen seit der Umsetzung von Basel III über die CRR/CRD IV (Capital Requirements Regulation Kapitaladäquanzverordnung/Capital Requirements Directive - Kapitaladäquanzrichtlinie) schon um 160 Milliarden Euro angewachsen ist und Berechnungen zufolge den Banken noch weitere 135 Milliarden Euro an Eigenkapital fehlen, um aktuelle und noch folgende Anforderungen zu erfüllen.

Daneben spielen hybride Finanzierungsinstrumente auch bei der Finanzierung von anderen Wirtschaftsunternehmen eine tragende Rolle. Um die aufgrund der Corona-Krise eingetretene schwierige Lage hinsichtlich der Liquidität auszugleichen, haben deutsche Unternehmen im ersten Halbjahr 2020 Unternehmensanleihen im Wert von $350 \mathrm{Mrd}$. Euro ausgegeben. Das ist bereits zu diesem frühen Zeitpunkt so viel, wie im Gesamtjahr 2018 emittiert wurde.

In den ersten beiden Kapiteln führt die Arbeit in die Grundlagen der Eigen- und Fremdkapitalfinanzierung ein, indem die Begriffe geklärt sowie Merkmale und Funktionen vorgestellt werden. Dabei wird insbesondere auf die Rolle der jeweiligen Kapitalklasse im Bereich der Finanzwirtschaft eingegangen. Finanzierung bedeutet dabei, dass einem Unternehmen Mittel zufließen, mit denen es eine Investition tätigen oder einen aktuellen Kapitalbedarf decken kann. Dafür werden im Gegenzug Finanzierungsinstrumente ausgegeben, bei denen es sich um ein durch ein Unternehmen geschaffenes Konstrukt handelt, dessen es sich bedient, um die Kapitalzufuhr zur Aufrechterhaltung oder Erweiterung seiner Tätigkeit zu ermöglichen. Die Finanzierung erfasst damit jede Art der Kapitalzufuhr, vom klassischen Darlehen bis zur Einlage eines Gesellschafters. Die Instrumente können dabei, je nach Bedürfnis des Emittenten und des Investors, die unterschiedlichsten Ausgestaltungen erfahren. Eine Einteilung in Eigen- oder Fremdkapital kann dann anhand der Ausgestaltungen immer nur für einen bestimmten Teilbereich erfolgen (HGB, Bankenaufsichtsrecht, Steuerrecht), unter Zugrundelegung der dort relevanten Normen und unter Berücksichtigung des jeweiligen Bilanzzwecks. Verglichen mit dem bilanziellen Eigenkapitalbegriff des Handelsrechts ist der bankaufsichtsrechtliche Eigenkapitalbegriff weiter gefasst. Darunter fallen neben Positionen, die schon nach der Handelsbilanz Eigenkapital darstellen, auch Passivpositionen, die handelsbilanzrechtlich als Fremdkapital verbucht werden. Ähnlichkeit besteht dagegen in der Funktion des Eigenkapitals als Haftungsgrundstock für die Verbindlichkeiten eines Unternehmens. Das Bankaufsichtsrecht dient der Sicherung der Funktionsfähigkeit des Finanzsystems und dem Schutz der Gläubiger jedes einzelnen Instituts. Dafür ist die Verlustausgleichsfunktion im laufenden Unternehmen von besonderer Bedeutung, dass es möglichst erst gar nicht zur Insolvenz eines Instituts kommt, was sich anschließend auf andere Unternehmen auswirken kann. 
Kapitel drei behandelt übersichtsartig die Entwicklung und den Inhalt bankaufsichtsrechtlicher Vorgaben. Von der allgemeinen Notwendigkeit der Regulierung von Banken sowie deren ökonomischen Funktionen wird zu der Entstehung und Entwicklung bankaufsichtsrechtlicher Vorgaben hingeführt, bevor die aus Basel I - III hervorgegangenen gesetzlichen Vorschriften näher beleuchtet werden. Den Abschluss des Kapitels bildet die Untersuchung, welche Kapitalklassen nach den aktuellen Regelungen von Basel III, der CRR bzw. CRD IV und dem KWG Anerkennung als bankaufsichtsrechtliches Eigenkapital finden können.

Im vierten Kapitel wird die besondere Stellung von hybriden Finanzierungsinstrumenten zwischen den Positionen Eigenkapital und Fremdkapital verdeutlicht. Die Arbeit nimmt eine Begriffsbestimmung vor und unternimmt den Versuch einer Klassifizierung.

Die Untersuchung einzelner hybrider Finanzierungsinstrumente erfolgt schließlich in Kapitel fünf der Arbeit. Dabei wird vor dem Hintergrund der Vielzahl an Ausgestaltungsformen eine Kategorisierung durchgeführt. Diese gliedert sich in anteilsbezogene Finanzierungsinstrumente, echte Hybridanleihen - hybride Finanzierungsinstrumente mit anteilsähnlichem Charakter, echte Hybridanleihen - hybride Fremdkapitalinstrumente, hybride Darlehen, sonstige hybride Finanzierungsinstrumente sowie Finanzierungsinstrumente unter Einsatz von SPVs. Die Arbeit beleuchtet die nahezu endlose Vielfalt an hybriden Finanzierungsinstrumenten, die nach den individuellen Bedürfnissen von Emittent und Investor ausgestaltet werden können. Ein besonderes Augenmerk liegt auf den unter der aktuellen bankenaufsichtsrechtlichen Regulierung anerkannten Finanzierungsinstrumenten, namentlich der bedingten Pflichtwandelanleihe und der Herabschreibungsanleihe. Dafür wird, ausgehend von der klassischen Wandelanleihe, deren Entstehung skizziert und der jeweilige Regulierungszusammenhang dargestellt. Neben theoretischen und rechtlichen Erwägungen geht die Arbeit immer wieder auf aktuelle Beispiele von Emissionen ein und beleuchtet deren Hintergrund.

Die Haupteigenschaft einer Wandelanleihe ist die Möglichkeit des Gläubigers, anstatt Rückzahlung in Geld die Wandlung in Aktien des Emittenten zu verlangen. Diese Wahlmöglichkeit stellt eine im Gesetz nicht geregelte, aber allgemein anerkannte Ersetzungsbefugnis dar. Zunächst hat der Gläubiger einen schuldrechtlichen Anspruch auf Zins- und Tilgungsleistung gegen den Emittenten. Wählt er die Wandlung, steht ihm - so die h.M. - erst ein schuldrechtlicher Anspruch auf Abschluss eines Zeichnungsvertrages über die Aktien zu. Macht daraufhin der Emittent ein den Anleihebedingungen entsprechendes Angebot, kann der Gläubiger dieses mit einer Bezugserklärung nach $\S 198$ Abs. 1 Satz 1 AktG annehmen. Mit dem Zeichnungsvertrag erhält der Emittent einen Anspruch auf Einlieferung der Schuldverschreibungen und der Gläubiger einen Anspruch auf Übertragung der Aktienrechte der Anleihen.

In Anbetracht der 2013 erfolgten Änderung der CRR und CRD IV hinsichtlich bedingter Pflichtwandelanleihen hat der deutsche Gesetzgeber mit der Aktienrechtsnovelle 2016 ausweislich der Regierungsbegründung dafür die umgekehrte Wandelanleihe normiert. Zwar wurde die Ausgabe schon vorher von der h.M. für möglich erachtet. Allerdings wollte der Gesetzgeber ausdrücklich klarstellen, dass dieses Finanzierungsinstrument unter $\S 221$ AktG und $\S 194$ Abs. 1 Satz 1 AktG (zeitlich gestreckte Bareinlage) fällt. Erreicht wurde dies, indem im Gesetzestext des § 221 Absatz 1 AktG die Wörter „oder der Gesellschaft“ eingefügt wurden. Danach konnte nun auch die Gesellschaft den Zeitpunkt der Wandlung bestimmen.

Rechtlich ergibt sich indes kein großer Unterschied zur klassischen Wandelanleihe. Die Ersetzungsbefugnis steht hier der Gesellschaft zu. Eine Besonderheit ergibt sich lediglich für die Wandlungserklärung: Da hier die Wandlungserklärung und Wandlungsentscheidung von der Gesellschaft ausgeht und diese dann wandeln wird, wenn es ihr am günstigsten erscheint, was möglicherweise nicht (immer) mit dem Willen des Investors übereinstimmt, bedarf es einer 
Ausgestaltung, die die Wandlung sicher eintreten lässt. Dafür hat sich in der Praxis die Gestaltung durchgesetzt, dass der Investor die Wandlungsstelle unwiderruflich dazu ermächtigt, die erforderliche Bezugserklärung für ihn abzugeben.

Mit der umgekehrten Wandelanleihe war somit die gesetzliche Grundlage für die nach der CRR erforderliche bedingte Pflichtwandelanleihe geschaffen. Zurückgehend auf einen Vorschlag der amerikanischen Ökonomen Culp und Flannery aus dem Jahr 2002 und auf Empfehlung des Baseler Ausschusses für Bankenaufsicht entschied sich der Europäische Gesetzgeber 2013 dazu, die bedingte Pflichtwandelanleihe in Art. 52 der Kapitaladäquanzverordnung aufzunehmen. Damit sollte den Aufsehern zufolge eine den Banken im Vergleich zur Ausgabe neuer Aktien günstigere Möglichkeit zur Verfügung stehen, ihr bankaufsichtsrechtliches Eigenkapital in Form von zusätzlichem Kernkapital zu erhöhen. Ziel der Konstruktion ist es, dass sich die Anleihe bei Erreichen einer bestimmten harten Kernkapitalquote des Unternehmens ,automatisch“ in Aktien wandelt. „Automatisch“ bedeutet dabei aber nicht automatisch im gewöhnlichen Sprachgebrauch, sondern es müssen ein paar Zwischenschritte erfolgen. Dies geschieht auch im Einklang mit dem Gesetz, wenn Art. 54 CRR davon spricht, dass die Wandlung „spätestens jedoch innerhalb eines Monats erfolgen soll“. Der Ablauf gestaltet sich dabei wie folgt: Zunächst wird bei Erreichen der kritischen Quote der Emittent die Wandlung erklären und ein den Anleihebedingungen entsprechendes Angebot zur Wandlung machen. Im Unterschied zur echten Bedingung aus $\S 158$ BGB, worauf der Name hindeutet, erfolgt der Eintritt nicht automatisch, da hier die genauen Bedingungen erst noch bestimmt werden müssen. Danach erklärt die Wandlungsstelle für den Gläubiger die Annahme, wodurch der Zeichnungsvertrag zustande kommt. Ab hier ist es der gleiche Ablauf wie bei der umgekehrten Wandelanleihe. Dadurch wird das bankaufsichtsrechtliche Eigenkapital direkt saniert, indem auf der Passivseite Schulden verringert und die geschaffenen Aktien nicht nur das bankaufsichtsrechtliche, sondern auch das nach HGB bilanzielle Eigenkapital erhöhen.

Die Herabschreibungsanleihe, die auch in Art. 52 der Kapitaladäquanzverordnung genannt ist, gestaltet sich - wie der Name schon andeutet - ein wenig anders. Hier schuldet der Emittent dem Gläubiger zunächst auch Zins und Tilgung wie bei einer gewöhnlichen Anleihe. Mit der Herabschreibung reduziert sich der geschuldete Betrag quotal um den erforderlichen Wert, damit die Quote des harten Kernkapitals wieder über den 5,125\% liegt. Rechtlich stellt dies eine Schuldreduzierung dar - Rückzahlungs- und Nennbetrag der Anleihe werden gleichermaßen reduziert. Der Gläubiger hat danach weiterhin einen schuldrechtlichen Anspruch auf Zinsleistungen eines eventuell verbleibenden Restbetrags. Herabschreibungsanleihen fallen nicht unter $\S 221 \mathrm{AktG}$, da der dahinterstehende Schutzzweck nicht berührt wird. Hauptvorteil für den Emittenten: keine Verwässerung und austarierte Stimmrechtsverhältnisse bleiben bestehen. Die Vorteile im Vergleich zur bedingten Pflichtwandelanleihe überwiegen wohl so sehr, dass Finanzinstitute in Deutschland hauptsächlich Herabschreibungsanleihen ausgegeben haben; das jedoch in sehr großem Ausmaß, was uns zu den systemischen Risiken der Instrumente führt.

Kapitel acht enthält die Untersuchung über das systemische Risiko von hybriden Finanzierungsinstrumenten. Systemisches Risiko bedeutet - vereinfacht gesagt - dass ein Risiko vorhanden ist, das sich bei Verwirklichung negativ auf die Funktionsweise wichtiger Teile oder des gesamten Finanz- und Wirtschaftssystems auswirkt. Darunter fallen einmal Makroschocks als systemisches Risiko im weiteren Sinn. Hier bilden Ursachen den Ausgangspunkt, deren Folgen viele oder alle Systemteilnehmer gleichzeitig treffen, ohne dass es erst einer Ansteckung bedarf - z.B. Zinsanstiege oder Währungsabwertungen. Auch darunter fallen Mikroschocks, bei denen die Ursache erst nur einen oder wenige Teilnehmer eines Systems betrifft, über Verbindungen aber andere Systemteilnehmer angesteckt werden.

Um die genaue Ansteckung im Finanzsystem nachzuvollziehen, behandelt die Arbeit kurz die ökonomische Funktion der Banken und die damit einhergehende Instituts- und 
Systeminstabilität. Die Banken erfüllen durch verschiedene Transformationsleistungen (Losgrößen-, Fristen- und Risikotransformation) wichtige Funktionen in einer Volkswirtschaft. Das hat aber auch zur Folge, dass das Bankengeschäft zwangsläufig einigen Unsicherheiten unterliegt. Dazu gehören etwa Aktivpositionen, die starken Kursschwankungen unterliegen, ein dem Geschäftsmodell innenliegender hoher Fremdkapitalanteil sowie die Abhängigkeit vom Vertrauen der Anleger. Zudem geht die Arbeit auf mögliche Ansteckungskanäle im Wirtschafts- und insbesondere im Finanzsystem ein. Eine direkte Ansteckung erfolgt, wenn ein Mikroschock durch unmittelbare Verbindungen in den Bilanzen auf andere Marktakteure übertragen wird - etwa wirkt sich die Zahlungsunfähigkeit eines Unternehmens über gehaltene Vermögenspositionen auf das Aktivvermögen eines anderen Unternehmens aus. Dieser Effekt der Ansteckung tritt durch die Globalisierung der Märkte und ihrer Akteure sowie die Globalisierung der Finanzinfrastruktur in den letzten Jahrzehnten immer häufiger auf.

Eine Besonderheit in der Finanzwirtschaft stellt die Praxis dar, überschüssige Mittel am Interbankenmarkt kurzfristig anzulegen. Kann eine Bank nun aber ihre kurzfristigen Verbindlichkeiten nicht revolvieren, wird sie zunächst diese Praxis einstellen. Andere Banken unternehmen den gleichen Schritt, um ihre Liquidität zu sichern, was letztendlich zu einem Versiegen der liquiden Mittel führt. Um trotz eines solchen „Liquiditätsschocks“ liquide zu bleiben, müssen die Banken Aktivvermögen veräußern (sog. fire sales), was durch das plötzliche Überangebot die Preise der Anlagewerte einbrechen lässt. Bei anderen Marktteilnehmern, die eine kontinuierliche Marktbewertung ihrer Aktiva vornehmen, führt dies zu steigenden Verlusten, was diese wiederum veranlasst, weitere Anlagewerte zu veräußern, was die Werte noch weiter sinken lässt und in eine Abwärtsspirale mündet. Für eine solche Ansteckung ist keine direkte Verbindung zwischen den Instituten erforderlich. Vielmehr vollzieht sich die Ansteckung auf indirektem Weg über das Marktverhalten. Zusätzlich tritt in der Finanzbranche die informationsbasierte Ansteckung hinzu. Dieser Ansteckungskanal resultiert allein aus dem ansteckenden Verhalten von Einlegern, die aufgrund asymmetrischer Informationsverteilung oder falscher Information unzureichend über Art und Ausmaß eines Schocks informiert sind und deshalb ihr Kapital abziehen. Weitet sich dieser Effekt aus, kann es schnell zu einem allgemeinen bank run kommen, bei dem Einleger ihrer Bank massenhaft Liquidität entziehen; dieser Effekt ist auch als Kapitalgeber-bank run möglich, wenn massenhaft Anleihen an der Börse veräußert werden. Der Bank bleibt in diesem Fall nur die Möglichkeit, kurzfristig Kapital aufzunehmen oder - falls dies nicht mehr möglich ist - weitere Vermögenswerte zu veräußern.

Nach der Abhandlung dieser allgemeinen Informationen geht die Untersuchung auf systemische Risiken von bedingten Pflichtwandel- und Herabschreibungsanleihen ein und zeigt zunächst auf, welche systemischen Risiken sich aus einer verschärften Regulierung allgemein ergeben, bevor einzelne Risikobereiche eingehend analysiert werden.

Problematisch im Rahmen des systemischen Risikos erscheint dabei, dass deutsche Finanzinstitute hauptsächlich Herabschreibungsanleihen begeben, bedingte Pflichtwandelanleihen dagegen kaum zum Einsatz kommen. Warum das mit Blick auf das systemische Risiko problematisch sein kann, hängt mit den Fehlanreizen zusammen, die Herabschreibungsanleihen und teilweise auch bedingte Pflichtwandelanleihen hervorrufen können. Findet eine Wandlung oder Herabschreibung statt, wird die Verschuldungsquote des Unternehmens reduziert. Das Fremdkapital verringert sich (im Fall der Wandelanleihe sogar zugunsten von hartem Kernkapital), und es führt zu einer höheren Eigenkapitalquote, was die Ausfallwahrscheinlichkeit wegen einer höheren Absorptionsfähigkeit verringert. Soweit der Grundgedanke. Allerdings bieten hybride Instrumente bei entsprechender Ausgestaltung auch Anreize, das Risiko zugunsten einer höheren Rendite zu erhöhen. Hat ein Institut Herabschreibungsanleihen ausgegeben und fällt ein Verlust an, tragen die Eigenkapitalgeber Verluste, solange die harte Kernkapitalquote über den in Art. 54 CRR festgeschriebenen 
5,125\% (oder einem höheren Prozentsatz liegt). Fällt die Quote darunter, wird der Forderungsbetrag der Anleihen herabgeschrieben und mit den Verlusten verrechnet. Dann tragen, sobald der Prozentsatz erreicht ist, erst die Anleihegläubiger anstatt der Eigenkapitalgeber die anfallenden Verluste. Dadurch findet sozusagen ein Vermögenstransfer von Anleihegläubigern zu Eigenkapitalgebern statt, was den Eigenkapitalgebern einen Anreiz geben könnte, ein höheres Risiko des Geschäfts $\mathrm{zu}$ veranlassen. Bei bedingten Pflichtwandelanleihen sind hingegen zwei Gestaltungen zu unterscheiden: verwässernde und nicht-verwässernde Wandelanleihen. Zunächst besteht eine ähnliche Situation wie bei Herabschreibungsanleihen: die Eigenkapitalgeber tragen anfallende Verluste, solange die harte Kernkapitalquote über den 5,125\% liegt. Fallen weitere Verluste an und fällt die harte Kernkapitalquote unter den Wert, tragen die bisherigen Eigenkapitalgeber mit den nach Wandlung neuen Eigenkapitalgebern gemeinsam die Verluste. Ob ein Anreiz besteht, die Gefahr auf sich zu nehmen, dass diese Situation eintritt, hängt vom jeweiligen Wandlungsverhältnis bzw. -preis ab. Schreiben die Anleihebedingungen ein hohes Wandlungsverhältnis vor, d.h. sind für eine Wandelanleihe eine Mehrzahl an Aktien zu liefern, oder einen niedrigen Wandlungspreis vor, d.h. ist für eine Aktie nur ein geringer Anleihebetrag erforderlich, ergibt sich eine verwässernde Wirkung für die Altaktionäre; sie tragen den Verlust mit den Neuaktionären gemeinsam und erfahren gleichzeitig aber eine Kapital- und Stimmrechtsverwässerung. Besteht ein niedriges Wandlungsverhältnis, d.h. sind für eine Aktie mehrere Wandelanleihen zu liefern, oder ein hoher Wandlungspreis, d.h. wird für eine Aktie ein hoher Anleihebetrag fällig, erfolgt keine hohe verwässernde Wirkung für die Altaktionäre. Vielmehr tragen sie sie tragen die Verluste gemeinsam, anschließend haben die neuen Aktionäre allerdings nicht so viele Rechte, wie ihnen bei einer $1 \mathrm{zu} 1$-Wandlung zustünden. Ein ungünstiges Wandlungsverhältnis bzw. ein ungünstiger Wandlungspreis bewirkt, dass die Anleihegläubiger im Fall einer Wandlung Teile ihrer Forderung verlieren, die dann zur Verlustabsorption genutzt wird. Deswegen besteht in diesem zweiten Fall für Altaktionäre der Anreiz, das Risiko auf Kosten der Anleihegläubiger zu erhöhen

Als weiteres Risiko lassen sich die sehr sensiblen Handlungspreise ausmachen. Als Folge der möglichen Vermögensverschiebung reagieren Handelspreise von bedingten Pflichtwandelanleihen und Herabschreibungsanleihen besonders volatil auf negative Nachrichten bezüglich der Finanzlage eines Instituts. Als im Februar 2016 Berichte aufkamen, die Deutsche Bank könne ihre Zinszahlungen auf CoCo-bonds wegen schlechter Ergebnisse aussetzen, hatte dies - trotz gegenteiliger Pressemitteilung - einen Kursrutsch nicht nur der Anleihe der Deutschen Bank, sondern auch der Anleihen vergleichbarer Emittenten zur Folge. Zudem zeigen Untersuchungen, dass Wandel- und Herabschreibungsanleihen bei sonst normaler Volatilität in Phasen sinkender Kurse überproportional an Wert verlieren - im Vergleich mit gewöhnlichen Anleihen gar bis zu fünf Mal so viel. Das deutet stark auf eine zusammenhängende Bewertung des Marktes durch Investoren hin und vergrößert die Gefahr, dass Kapitalanleger das Vertrauen in diesen Anlagebereich verlieren, sollte eine Bank gezwungen sein, ihre Anleihen zu wandeln oder herabzuschreiben.

Systemisches Risiko kann darüber hinaus auch darin bestehen, dass diese nach außen einheitlich wirkenden Instrumente inhaltlich ganz individuellen Ausgestaltungen unterliegen. Selbst unter den europäischen Regelungen bleibt Art. 54 CRR, der mit 5,125\% harter Kernkapitalquote den spätesten Punkt einer Wandlung bzw. Herabschreibung beziffert, die einzige Konstante. Darüber hinaus besteht keine weitere Standardisierung. Höhe und Anzahl der Auslöseereignisse, genaue Ausgestaltung von Umwandlungsmechanismen, erforderliche Kapitalpuffer, vorübergehende oder dauerhafte Herabschreibung, genaue Verlustabsorption, Häufigkeit von Zinszahlungen etc. bleiben der Ausgestaltung der Parteien vorbehalten. Das erschwert eine angemessene Risikobewertung und führt über verringertes Vertrauen der Systemteilnehmer zu Unsicherheiten im Finanzsystem. 
Mit diesem Problem verbunden ist die unzureichende Übersicht über die genauen Risikopositionen, die sich aus der Komplexität und Verschiedenheit der Produkte ergibt. Da die Zuverlässigkeit mathematischer Finanzmodelle zur Risikobewertung bei zunehmender Komplexität nachlässt, greifen viele Anleger auf externe Bewertungen zurück, um das Risiko von Finanzanlagen einzuschätzen. Dies kann aber zur Verunsicherung über das Ausfallrisiko der Gegenpartei führen, wenn aufgrund des Verhaltens anderer Investoren gehandelt wird. Im Vorfeld der Finanzkrise 2007/08 wurde solches Herdenverhalten dadurch dokumentiert, dass Investoren falschen Informationen über den wahren Wert gehebelter Asset Backed Securities folgten. Gleiches kann auch für die vorliegenden Instrumente gelten, wenn ControllingAbteilungen keine Übersicht mehr haben, welchem Risiko das Unternehmen durch welche Produkte ausgesetzt ist, und sie bei einem negativen Ereignis ohne tiefere Analyse anderen Marktteilnehmern mit dem Verkauf betroffener Aktiva folgen. 